Measur enent of der i vat $i$ ve of $i$ on temper at ure using hi gh spatial resol uti on charge exchange spect r oscopy wi th space modul at i on opt i cs

\begin{tabular}{|l|l|}
\hline $\begin{array}{l}\text { j our nal or } \\
\text { publ i cat i on t i t l e }\end{array}$ & Revi ew of Sci ent i f i c I nst r ument s \\
\hline vol une & Vol . 79 \\
\hline page r ange & 053506- - 053506- 6 \\
\hline year & 2008- 05-01 \\
\hline URL & ht t p: //hdl . handl e. net /10655/3814 \\
\hline
\end{tabular}




\title{
Measurement of derivative of ion temperature using high spatial resolution charge exchange spectroscopy with space modulation optics
}

\author{
K. Ida, ${ }^{1}$ Y. Sakamoto, ${ }^{2}$ M. Yoshinuma, ${ }^{1}$ S. Inagaki, ${ }^{1}$ T. Kobuchi, ${ }^{1}$ G. Matsunaga,${ }^{2}$ and \\ Y. Koide ${ }^{2}$ \\ ${ }^{1}$ National Institute for Fusion Science, Toki 509-5292, Japan \\ ${ }^{2}$ Japan Atomic Energy Agency, Naka, Ibaraki-ken 311-0193, Japan
}

(Received 24 June 2007; accepted 16 April 2008; published online 23 May 2008)

\begin{abstract}
A new technique to measure the first and second derivatives of the ion temperature profile has been developed by using a charge exchange spectroscopy system with space modulation optics. The space observed is scanned up to $\pm 3 \mathrm{~cm}$ with a cosine wave modulation frequency up to $30 \mathrm{~Hz}$ by shifting the object lens in front of the optical fiber bundle by $0.5 \mathrm{~mm}$ with a piezoelement. The first and second derivatives of ion temperature are derived from the modulation component of the ion temperature measured by using Fourier series expansion. () 2008 American Institute of Physics.

[DOI: $10.1063 / 1.2921702]$
\end{abstract}

\section{INTRODUCTION}

Charge exchange spectroscopy has been widely used as a powerful tool to measure radial profiles of ion temperature in tokamak and stellarators because it gives many observation chords through the advantage of optical fiber arrays. ${ }^{1-6}$ The charge exchange collisions between fully ionized carbon $\left(\mathrm{C}^{6+}\right)$ and hydrogen or deuterium atoms in a heating neutral beam result in an excited ion with one electron $\left(\mathrm{C}^{5+}\right)$. The ion temperature is inferred from the Doppler broadening of the spectral line of $\mathrm{C}$ VI $(n=8 \rightarrow 7)$. The ion temperature profiles measured with charge exchange spectroscopy are also used to evaluate the first derivative of the ion temperature profile (temperature gradient) to calculate the ion thermal diffusivity in the heat transport.

The uncertainties of the ion temperature gradient determined by the temperature values at adjacent chords are typically large $(>20 \%)$; therefore, the error bar of the thermal diffusivity is mainly determined by the uncertainty of the measured temperature gradient. The fitting curve is often used to reduce the uncertainties of the ion temperature gradient. This approach is useful for the plasma in $L$ mode, where the variation of the temperature gradient is small enough to fit the ion temperature profile with a relatively small number of the fitting parameters such as a polynomial function $\left(T_{0}+T_{1} \rho^{2}+T_{2} \rho^{4}+T_{3} \rho^{6}+\ldots\right)$. However, fitting with a polynomial function has problems for the plasma with an internal transport barrier (ITB), which is observed in tokamaks ${ }^{7-14}$ and helical systems, ${ }^{15,16}$ because the polynomial function does not fit the ion temperature profiles observed with a significant variation of temperature gradient in space. In these plasmas, the temperature gradient evaluated from the fitting curves depends on which fitting function is chosen. In spite of the importance of evaluating the temperature gradient, there is no direct measurement of temperature gradient (first derivative of temperature) proposed. In this paper, the direct measurement of first and second derivative ion temperature profiles are discussed.

\section{EXPERIMENTAL SETUP OF HIGH SPATIAL RESOLUTION CHARGE EXCHANGE SPECTROSCOPY}

To evaluate the temperature gradient, the error bar of the ion temperature should be less than $(a / n) \partial T_{i} / \partial r$, where $a$ is the plasma minor radius and $n$ is the spatial resolution of the measurement of the temperature gradient. The requirement for the error bar of ion temperature measurements depends on the temperature gradient and spatial resolution. The relative error bar should be a few percent when the spatial resolution $n$ is $20-30$. The number of photons is a key parameter to determine the error bar due to the photon statics; therefore, a high throughput of the spectroscopy system is required. The high throughput of the spectroscopy system has been developed to measure the temperature gradient.

Figure 1 shows the experimental setup of high spatial resolution charge exchange spectroscopy. The light of the charge exchange line is led to the entrance of the CzernyTurner spectrometer with a $f=400 \mathrm{~mm} \mathrm{~F} 2.8$ camera lens and a $2160 / \mathrm{mm}$ grating (Bunkou-Keiki CLP-400) using a 32 channel optical fiber array. One of the channels of the optical fiber array is connected to a samarium lamp for wavelength calibration, and 31 channels of the optical fiber array are connected to the object optical system for the measurements. The diameter of the core is $200 \mu \mathrm{m}$ and the diameter of the cladding is $250 \mu \mathrm{m}$. There are dummy fibers between the channels arranged to make room for scanning and the distance between adjacent chords is $500 \mu \mathrm{m}$.

The back illuminated electron multiplying 512 $\times 512$ pixel $\left(16 \times 16 \mu \mathrm{m}^{2}\right)$ charge coupled device $(\mathrm{CCD})$ camera (Andor DV887DCS-BV) is used as a detector to achieve a high frame rate $f_{\text {ccd }}$, up to $400 \mathrm{~Hz}$ with 16 pixel vertical binning. To match the image of the optical fiber into the strip of the CCD $(256 \mu \mathrm{m})$, the optical fiber array is arranged at the slit with a pitch control of $256 \pm 20 \mu \mathrm{m}$. The object lens $(f=20 \mathrm{~mm})$ in front of the fiber array is mounted on the long-range Piezo nanotranslation stage (Physik Instrumente P-625.1CD), which has a travel range of $500 \mu \mathrm{m}$. In general, either object lens or fiber bundle can be shifted to 


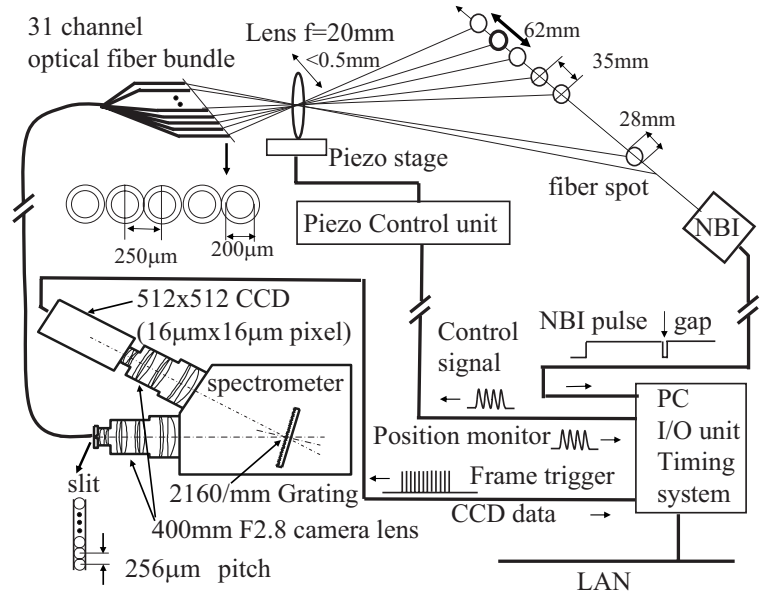

FIG. 1. Experimental setup for high spatial resolution charge exchange spectroscopy.

scan the observation point in the plasma. Since the frequency of the scan of the Piezo stage depends on the load, the object lens, which has smaller mass $(63 \mathrm{~g})$ than that of the fiber bundle, is scanned. The observation point is scanned by $\pm 31 \mathrm{~mm}$ (corresponding to $\pm 24 \mathrm{~mm}$ in radius). Because the distance between two chords is $36 \mathrm{~mm}$, the observation points overlap during the scan, while the diameter of the spot size of the image of the optical fiber in the plasma $d$ is $28 \mathrm{~mm}$.

Figure 2 shows the full width of scan as a function of scan frequency and the voltage applied to the Piezo control unit (E-610, position servocontrol board with amplifier with a gain factor of 10). The full width of the scan at the cross section of the plasma where the neutral beam is injected is roughly proportional to the voltage applied to the controller
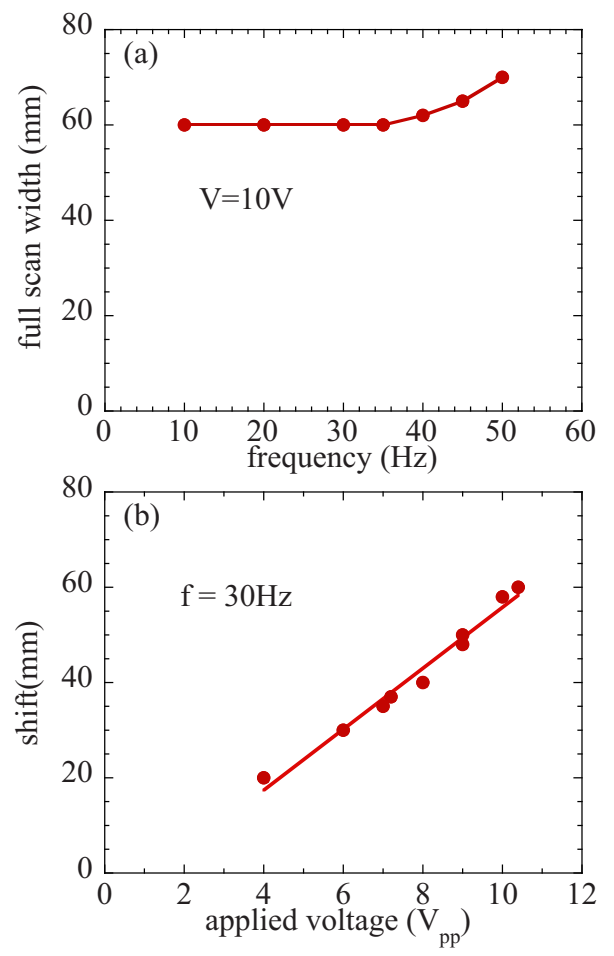

FIG. 2. (Color online) Full scan width of space modulation optics as a function of (a) frequency and (b) applied voltage.

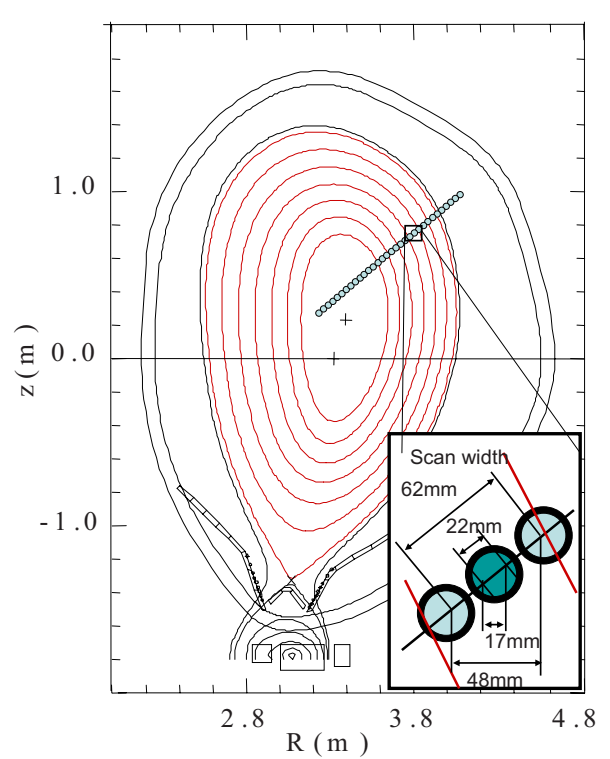

FIG. 3. (Color online) Poloidal cross section of JT-60U plasma. The spots of optical fiber are also plotted.

and it becomes $60 \mathrm{~mm}$ at a voltage of $10 \mathrm{~V}$ (voltage applied to the Piezo is $100 \mathrm{~V}$ ). The full width of the scan starts to increase when the scanning frequency $f_{m}$ exceeds $35 \mathrm{~Hz}$, which is one-third of the resonance frequency for the load in this setup. Because of the inertia of Piezo stage, lens mount, and lens, there is a delay of the actual travel from the control voltage. In our experimental setup, the delay time is $5 \mathrm{~ms}$, which is taken into account in the analysis.

There is a trade-off between the frame rate of the CCD $f_{\text {ccd }}$ and the error bar of the ion temperature measured because a larger photon number due to a larger accumulation time is necessary to reduce the error bar of the measurement. As the modulation frequency $f_{m}$ is increased, the number of spatial data points measured can be increased. However, there is a minimum value of the spatial resolution $d_{s}$ determined by the spot size of the image, which is simply given as $(\pi / 4) d$, by averaging the area of image. The spatial resolution determined by the modulation speed and sampling speed $d_{m}$ can be expressed as $\left(w_{s} / 2\right) \sin (\omega t) 2 \pi f_{m} / f_{\text {ccd }}$, where $w_{s}$ is the full width of scan. The spatial resolution changes in time during the modulation, and the maximum of the spatial resolution $d_{m}$ is $\pi w_{s} f_{m} / f_{\text {ccd }}$. The maximum of the spatial resolution during the modulation, $d_{m}$, should be smaller than the radial resolution determined by the spot size of the image of optical fiber, $d_{s}$, to eliminate the gap in the measurements.

As seen in the cross section of JT-60U in Fig. 3, the radial profiles of ion temperature are measured from the plasma edge to the plasma center along the beamline with 31 observation points. The observation points are scanned along the beamline with a full width of $62 \mathrm{~mm}$, which corresponds to the radial scan with $48 \mathrm{~mm}$, by using the modulation optics of the charge exchange spectroscopy. In this experiment, by choosing $f_{\text {ccd }}=200 \mathrm{~Hz}$ and $f_{m}=10 \mathrm{~Hz}$, the modulation charge exchange spectroscopy provides the radial profile of ion temperature with 310 points every $50 \mathrm{~ms}$. The maximum spatial resolution determined by the modulation frequency, 

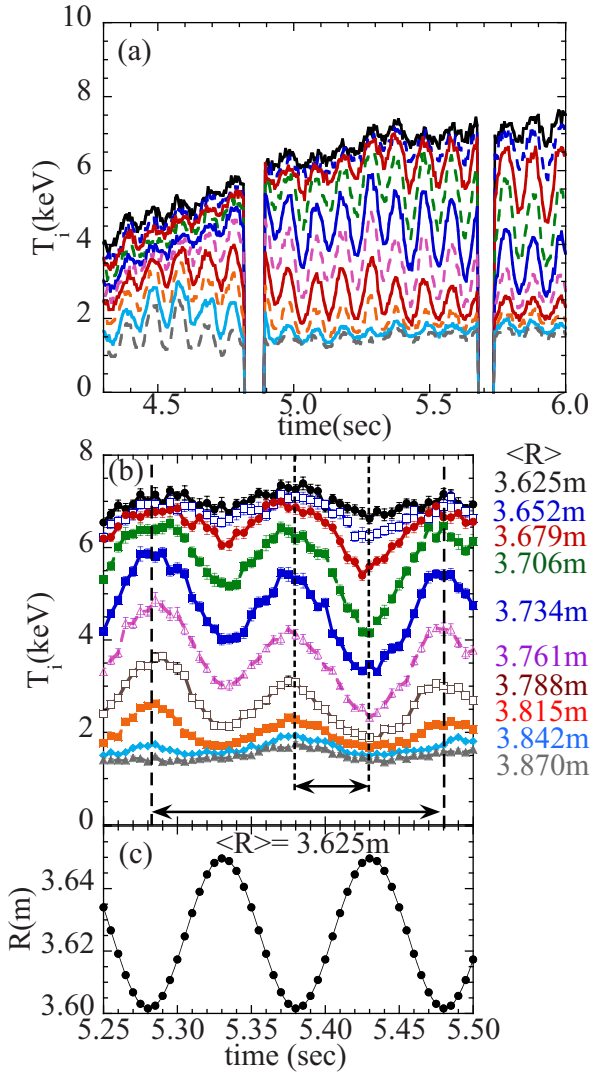

FIG. 4. (Color online) Time evolution of ion temperature measured with high spatial resolution charge exchange spectroscopy with space modulation optics at various radius for $t=4.5-7.0$ and $5.25-5.50 \mathrm{~s}$ (expanded view).

$d_{m}$, is $10 \mathrm{~mm}$ and the spatial resolution determined by the spot size, $d_{s}$, is $22 \mathrm{~mm}$, which corresponds to the radial resolution of $17 \mathrm{~mm}$.

The time evolution of the ion temperature measured is modulated by a cosine wave, as seen in Fig. 4. The time evolution of the ion temperature and the major radius of the measurement in the expanded time scale are plotted in Figs. 4(b) and 4(c), respectively. The amplitude of the modulation is proportional to the magnitude of the temperature gradient; the modulation amplitude becomes large at the ITB region. To subtract the background emission due to the charge exchange reaction between the fully ionized impurity and the thermal neutral at the plasma periphery (cold component), the neutral beam is turned off in a short time of $70 \mathrm{~ms}$ (neutral beam injection gap). The background emission between the two gaps is calculated by interpolating the background emission at the gaps. The cold component does not have a modulation component because the cold component is more or less constant in space. The amplitude of the modulation of the ion temperature is significant at the time averaged major radius $\langle R\rangle$ of 3.67-3.81 $\mathrm{m}$, where the temperature gradient becomes large due to the existence of an ITB. By scanning the observation point with modulation optics, the radial points of the measurement can be multiplied by $M$ $=f_{\text {ccd }} /\left(2 f_{m}\right)$ if the plasma is in steady state.

Figure 5 shows an example of a radial profile of ion temperature derived from the modulation period of $5.385-5.435 \mathrm{~s}$, which is indicated in Fig. 4(b). The radial profile of ion temperature is fitted by using a modified hy-

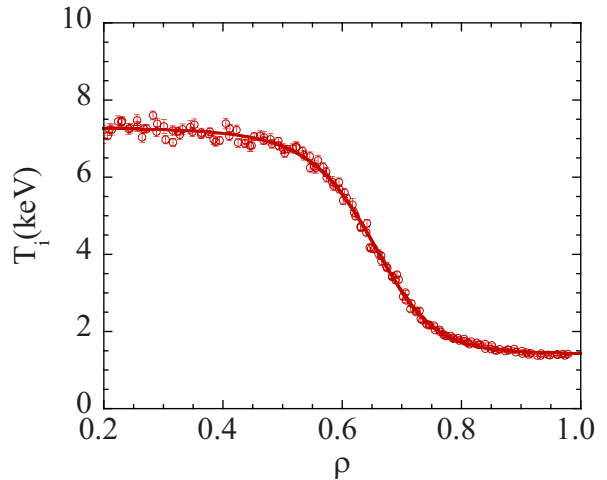

FIG. 5. (Color online) Radial profile of ion temperature derived from the time evolution of ion temperature by scanning the radius observed by $48 \mathrm{~mm}$ at $t=5.385-5.435 \mathrm{~s}$. The fitting curve using a modified hyperbolic tangent $\alpha \tanh \left[\left(R-R_{1}\right)^{2} / w\right]-\beta\left(R-R_{2}\right)$ is plotted with line.

perbolic tangent $\alpha \tanh \left[\left(R-R_{1}\right)^{2} / w\right]-\beta\left(R-R_{2}\right)$, which is often used to represent the radial profile of temperature at the transport barrier. The error tends to become larger toward the plasma center (the magnetic axis is at $R=3.4 \mathrm{~m}$ ), where the neutral beam for the charge exchange spectroscopy attenuates and the signal becomes weak. The overall shape of the fitting curve is good enough to represent the radial profile of the ion temperature but may not be good enough to discuss the first and second derivatives of the ion temperature profiles by taking a derivative of the fitting curve. The radial profiles of the first and second derivatives strongly depend on the function that is used for the fitting. Therefore, it is desirable to measure the temperature gradient without using the fitting curve. In the next section, the direct measurement of the first and second derivatives of the ion temperature profiles by using Fourier series expansion is discussed.

\section{FOURIER SERIES EXPANSION}

When there is no curvature of the temperature profile $\left(\partial^{2} T_{i} / \partial r^{2}=0\right)$ and the temperature profile has a linear slope, since the radial position of each observation point $R$ changes in time with a cosine wave as $\delta r=\Delta r \cos (\omega t), \omega=2 \pi / f_{\bmod }$, the time evolution of the ion temperature also becomes a cosine wave as $T_{i}(t)=T_{i}(0)+\Delta T_{i} \cos (\omega t)$. However, if there is a curvature in the temperature profile, the time evolution of the ion temperature has higher harmonic terms and the time evolution of the ion temperature $T_{i}(t)$ can be expressed as

$$
\begin{aligned}
& T_{i}(t)=a_{0}+a_{1} \cos (\omega t)+a_{2} \cos (2 \omega t)+\cdots, \\
& a_{0}=\frac{1}{\tau} \int_{0}^{\tau} T_{i}(t) d t, \\
& a_{n}=\frac{2}{\tau} \int_{0}^{\tau} T_{i}(t) \cos (n \omega t) d t .
\end{aligned}
$$

By using $\Delta r$ and Fourier series coefficients $a_{n}$, the first and second derivatives of ion temperature profiles are given as

$$
\frac{\partial T_{i}(r)}{\partial r}=\frac{a_{1}}{\Delta r}
$$




$$
\frac{\partial^{2} T_{i}(r)}{\partial r^{2}}=\frac{4 a_{2}}{(\Delta r)^{2}} .
$$

The first and second derivatives of the ion temperature can be derived by integrating $T_{i}(t) \cos (\omega t)$ and $T_{i}(t) \cos (2 \omega t)$ over one (or a few) period(s) of the cosine wave. The error bar of the first and second derivatives can be evaluated from the error bar of the ion temperature measured by statistical analysis. The ion temperature with an error bar of standard deviation of $\Delta T_{i}(t)$ can be expressed statistically as

$$
T_{i}^{j}(t)=T_{i}(t)+\Delta T_{i}(t) g_{r}^{j},
$$

where $g_{r}^{j}$ is a random number which satisfies $\left\langle g_{r}^{j}\right\rangle=0$ and $\left\langle\left(g_{r}^{j}\right)^{2}\right\rangle=1$. The ion temperature $T_{i}(t)$ and its error bar $\Delta T_{i}(t)$ are calculated from the Gaussian fitting of the Doppler width (and its error bar) of the line emission (at $529.05 \mathrm{~nm}$ ) between fully ionized carbon and the neutral beam. The error bars of the ion temperature consist of the statistical error from fitting the Gaussian and the uncertainty in the base line. ${ }^{4}$ The magnitude of the error bars depends on the number of photons and the noise of the CCD detector. The error bar of the Fourier coefficient can be given with a statistical treatments. ${ }^{17}$ The transfer of the error bar of the ion temperature to the error bar of a coefficient $a_{n}$ can be simply calculated by the statistical approach, and the error bar of the coefficient $a_{n}$ is given by

$$
\Delta a_{n}=\sqrt{\frac{1}{N} \sum_{j=1}^{N}\left[a_{n}^{j}-a_{n}\right]^{2}}
$$

where

$$
a_{n}^{j}=\frac{2}{\tau} \int_{0}^{\tau} T_{i}^{j}(t) \cos (n \omega t) d t .
$$

Here, $N$ is the number of random error generation that should be large enough to derive error bars and is taken to be 256 in this analysis. The error bar of first and second derivatives of the temperature profile are given by $\Delta a_{1} /(\Delta r)$ and $4 \Delta a_{2} /(\Delta r)^{2}$. As an alternative technique, the first and second derivatives of the ion temperature profiles can be evaluated by using a simple Taylor series expansion as ${ }^{18}$

$$
T_{i}(R)=T_{i}\left(R_{0}\right)+\frac{\partial T_{i}(r)}{\partial r} \delta r+\frac{\partial^{2} T_{i}(r)}{2 \partial r^{2}}(\delta r)^{2}+\cdots,
$$

where $\delta r=R-R_{0}$ and $R_{0}$ is the measurement position in the plasma without modulation. The first and second derivatives of the ion temperature profiles and their error bars are also obtained from the data for each chord $\left(R_{0}\right)$ by using a linear least-squares fit to the polynomial function of $\delta r$. There are two significant advantages of a Fourier or Taylor series expansion compared to the analysis using a curve fitting. One is that no assumption of the function of the fitting curve is necessary and the other is that this technique has a straightforward estimation of the error bar of the derivative, which is complicated and also depends on the assumption of the function in the curve fitting. When the number of cycles of the cosine oscillation is small, the Taylor series expansion seems to be better than the Fourier series expansion because the Fourier series expansion is sensitive to the boundary condi-
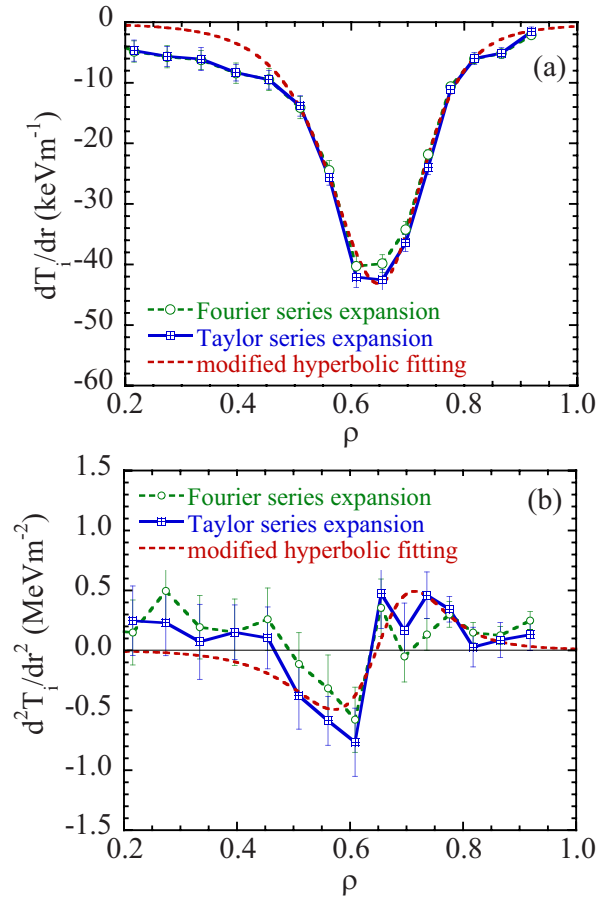

FIG. 6. (Color online) Radial profiles of the (a) first and (b) second derivatives of ion temperature evaluated by the Taylor and Fourier series expansions. The dashed lines are the first and second derivatives derived from the fitting curve with a modified hyperbolic tangent.

tion. However, as the number of cycles of the cosine oscillation is increased, the Fourier series expansion has an advantage in eliminating a gradual change in the ion temperature slower than the cycle of the cosine oscillation.

Figure 6 shows the radial profiles of the first and second derivatives of the ion temperature profiles derived from the one period of modulation at $t=5.38-5.48 \mathrm{~s}$ in Fig. 4(b). The first and second derivatives derived from the Taylor and Fourier series expansions and fitting curve with modified hyperbolic tangent are also plotted for comparison. The radial profile of the first derivative of ion temperature has a peak of $-40 \mathrm{keV} / \mathrm{m}$ at the middle of internal transport barrier $(R$ $=3.7 \mathrm{~m}$ ). The magnitude of the gradient is roughly consistent with the result of the curve fitting. However, there are significant differences in the radial profile of the first derivative at $\rho=0.2-0.5$ between the Taylor and Fourier series expansions and the curve fitting with modified hyperbolic tangent. The radial profile of the first derivative derived from curve fitting with a modified hyperbolic tangent is symmetric, but the first derivative derived from the Taylor and Fourier series expansions has an asymmetric profile. This is because the modified hyperbolic tangent function is not good enough to represent the real radial profile of the first derivative of the ion temperature profile. There are also differences in the radial profile of the second derivative at $\rho=0.2-0.5$, as seen in Fig. 6(b). It should be pointed out that the modified hyperbolic tangent function always gives a symmetric profile of the second derivative of the ion temperature because of the characteristics of the function itself.

In general, an increase in the number of cycles of the cosine oscillation contributes to the improvement of the accuracy of the first and second derivatives of the ion tempera- 

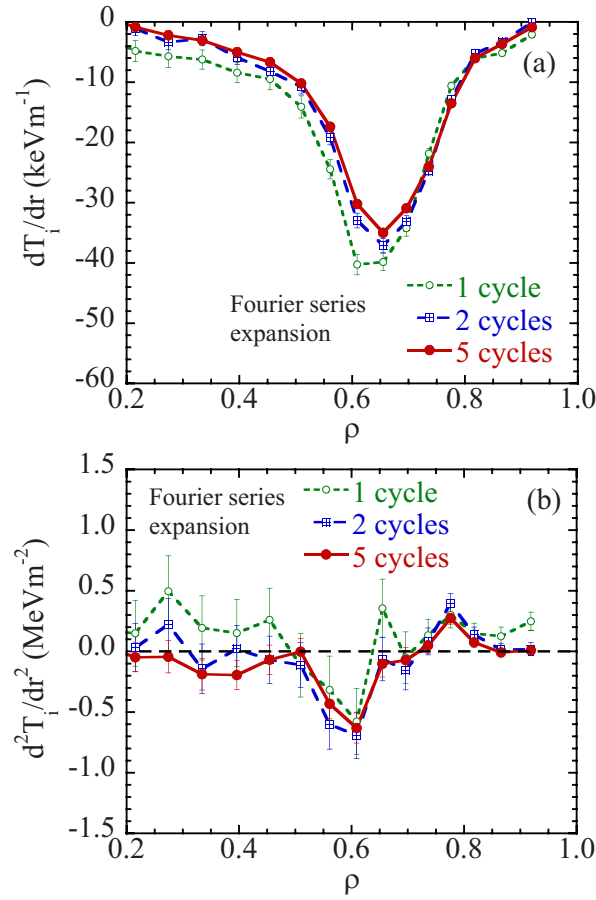

FIG. 7. (Color online) Radial profiles of the (a) first and (b) second derivatives of ion temperature evaluated by Fourier analysis with 1 cycle, 2 cycles, and 5 cycles of the cosine oscillation.

ture profile in doing the Fourier analysis. The number of cosine oscillations for Fourier analysis should be minimized to improve the time resolution of the measurements. Therefore, the number of cycles depends on the quality of the ion temperature measurements and how the plasma is in steady state. In Fig. 7, the first and second derivatives of the ion temperature profile with various numbers of cycles are plotted. Although the error bars are relatively large in the data derived with one cycle, the error bars in the data with two and five cycles are small enough to discuss the radial structure of the first and second derivatives of the ion temperature profiles. In this experiment, two and five cycle integrations are considered to be enough to give radial profiles of the first derivative and second derivatives of the ion temperature profile, respectively. It should be noted that the lack of antisymmetric radial profiles in the second derivative of the ion temperature profile becomes clearer as the number of cycles of the cosine oscillation is increased and it is due to plasma physics not due to nonlinearities in the system affecting the second harmonic term.

\section{DISCUSSION}

The second harmonic term of the modulation cosine wave may cause a systematic error in the computation of the second derivative. The motion of the translation stage is measured with a position monitor signal of the long-range Piezo nanotranslation stage. The second harmonic term of the modulated location in the plasma is only $4 \times 10^{-3}$ of the primary term. Since the linear motion of the translation stage produces the linear motion of the spot measured in the plasma, optical aberrations do not cause a systematic error in the computation of the second derivative. A new technique to measure the first and second derivatives of the ion temperature profile has been developed by using the charge exchange spectroscopy system with space modulation optics. This technique gives a direct measurement of the first and second derivatives of ion temperature, which does not require any assumption of a fitting function, which causes significant ambiguity in the estimates of the first and second derivatives. For example, the modified hyperbolic tangent often used to represent the radial profile of the ion temperature at the transport barrier cannot represent the asymmetric profile of the second derivative. In this analysis, Fourier components with higher order $a_{n}(n \geqslant 3)$ are ignored because the error bar becomes too large to discuss the higher derivative. Because of this neglect of the higher order Fourier components, the radial profile of the $n$th derivative is not identical to the integrated $(n+1)$ th derivative profile. For example, the first derivative obtained from the Fourier component with Eq. (4) is not identical to that derived from the integration of the radial profile of second derivative given by Eq. (5).

In these measurements, the first and second derivatives of ion temperature are derived from two periods of radial scan. When the plasma is in steady state for a longer time, the accuracy of the measurements can be improved by increasing the integrating period. In conclusion, this technique will provide a significant impact in the transport analysis because the temperature gradient and its error bar are directly derived. Moreover, the radial profiles of the second derivative of ion temperature profiles measured would contribute to the study of the detailed structure and mechanism of an ITB plasma.

\section{ACKNOWLEDGMENTS}

We would like to thank the JT-60 Team for their effort to support the experiment in JT-60U. This work is partly supported by a Grant-in-aid for Scientific Research (No. 18206094) and the Grant-in-Aid for Specially-Promoted Research (No. 17044010) of MEXT Japan.

${ }^{1}$ R. J. Fonck, M. Finkenthal, R. J. Goldston, D. L. Herndon, R. A. Hulse, R. Kaita, and D. D. Meyerhofer, Phys. Rev. Lett. 49, 737 (1982).

${ }^{2}$ R. J. Groebner, N. H. Brooks, K. H. Burrell, and L. Pottler, Appl. Phys. Lett. 43, 920 (1983).

${ }^{3}$ R. J. Fonck, D. S. Darrow, and K. P. Jaehnig, Phys. Rev. A 29, 3288 (1984).

${ }^{4}$ K. Ida and S. Hidekuma, Rev. Sci. Instrum. 60, 867 (1989).

${ }^{5}$ R. C. Isler, Plasma Phys. Controlled Fusion 36, 171 (1994).

${ }^{6}$ K. Ida, S. Kado, and Y. Liang, Rev. Sci. Instrum. 71, 2360 (2000).

${ }^{7}$ Y. Koide, M. Kikuchi, M. Mori, S. Tsuji, S. Ishida, N. Asakura, Y. Kamada, T. Nishitani, Y. Kawano, T. Hatae, T. Fujita, T. Fukuda, A. Sakasai, T. Kondoh, R. Yoshino, and Y. Neyatani, Phys. Rev. Lett. 72, 3662 (1994).

${ }^{8}$ T. Fujita, S. Ide, H. Shirai, M. Kikuchi, O. Naito, Y. Koide, S. Takeji, H. Kubo, and S. Ishida, Phys. Rev. Lett. 78, 2377 (1997).

${ }^{9}$ O. Gruber, R. C. Wolf, R. Dux, C. Fuchs, S. Gunter, A. Kallenbach, K. Lackner, M. Maraschek, P. J. McCarthy, H. Meister, G. Pereverzev, F. Ryter, J. Schweinzer, U. Seidel, S. Sesnic, A. Stabler, J. Stober, and the ASDEX Upgrade Team, Phys. Rev. Lett. 83, 1787 (1999).

${ }^{10}$ T. J. J. Tala, J. A. Heikkinen, V. V. Parail, Yu. F. Baranov, and S. J. Karttunen, Plasma Phys. Controlled Fusion 43, 507 (2001).

${ }^{11}$ J. E. Rice, R. L. Boivin, P. T. Bonoli, J. A. Goetz, R. S. Granetz, M. J. Greenwald, I. H. Hutchinson, E. S. Marmar, G. Schilling, J. A. Snipes, S. M. Wolfe, S. J. Wukitch, C. L. Fiore, J. H. Irby, D. Mossessian, and M. Porkolab, Nucl. Fusion 41, 277 (2001).

${ }^{12}$ Y. Sakamoto, Y. Kamada, S. Ide, T. Fujita, H. Shirai, T. Takizuka, Y. Koide, T. Fukuda, T. Oikawa, T. Suzuki, K. Shinohara, R. Yoshino, and 
JT-60 Team, Nucl. Fusion 41, 865 (2001).

${ }^{13}$ E. J. Doyle, C. M. Greenfield, M. E. Austin, L. R. Baylor, K. H. Burrell, T. A. Casper, J. C. DeBoo, D. R. Ernst, C. Fenzi, P. Gohil, R. J. Groebner, W. W. Heidbrink, G. L. Jackson, T. C. Jernigan, J. E. Kinsey, L. L. Lao, M. Makowski, G. R. McKee, M. Murakami, W. A. Peebles1, M. Porkolab, R. Prater, C. L. Rettig, T. L. Rhodes, J. C. Rost, G. M. Staebler, B. W. Stallard, E. J. Strait, E. J. Synakowski, D. M. Thomas, M. R. Wade, R. E. Waltz, and L. Zeng, Nucl. Fusion 42, 333 (2002).

${ }^{14}$ H. Takenaga, N. Oyama, L. G. Bruskin, A. Mase, T. Takizuka, and T. Fujita, Plasma Phys. Controlled Fusion 48, A401 (2006).
${ }^{15}$ U. Stroth, K. Itoh, S.-I. Itoh, H. Hartfuss1, H. Laqua1, the ECRH Team 1, and the W7-AS Team 1, Phys. Rev. Lett. 86, 5910 (2001).

${ }^{16}$ K. Ida, T. Shimozuma, H. Funaba, K. Narihara, S. Kubo, S. Murakami, A. Wakasa, M. Yokoyama, Y. Takeiri, K. Y. Watanabe, K. Tanaka, M. Yoshinuma, Y. Liang, N. Ohyabu, and LHD Experimental Group, Phys. Rev. Lett. 91, 085003 (2003).

${ }^{17}$ J. R. Taylor, An Introduction to Error Analysis: The study of Uncertainties in Physical Measurements (University Science Books, Sausalito, 1997).

${ }^{18}$ P. R. Bevington and D. K. Robinson, Data Reduction and Error Analysis for the Physical Sciences (McGraw-Hill, New York, 1991). 